\title{
COMPETENCIAS GERENCIALES DE LOS EMPRESARIOS Y SU RELACIÓN CON LOS FACTORES QUE LIMITAN EL CRECIMIENTO EMPRESARIAL DE LAS MYPES AGROEXPORTADORAS DE ACEITUNA Y DERIVADOS DE TACNA.
}

\begin{abstract}
BUSINESS MANAGEMENT COMPETENCES AND THEIR RELATIONSHIP WITH THE FACTORS THAT LIMIT THE BUSINESS GROWTH OF AGROEXPORTING MYPES OF OLIVE AND DERIVATIVES OF TACNA. 2018.
\end{abstract}

\author{
Dennys Sihuayro Larico ${ }^{1}$ \\ Efren Eugenio Chaparro Montoya²
}

Aceptado: $17 / 09 / 2019$

Publicado online:15/01/2020

\begin{abstract}
RESUMEN
Se tuvo como objetivo determinar la relación que existe entre las competencias gerenciales de los empresarios y los factores que limitan el crecimiento empresarial de las MYPES agroexportadoras de aceituna y derivados de Tacna. La investigación correlacional de corte transversal. De acuerdo con la interferencia del investigador en el estudio, es una investigación no experimental. La población está delimitada a los empresarios MYPE dedicadas al rubro agroexportadoras de aceituna y derivados de la ciudad de Tacna, el tamaño de muestra seleccionado aplicando el muestreo no probabilístico. El procesamiento de los datos implicó Tablas de frecuencias, gráficos de barras, pruebas de asociación Chi cuadrado y el coeficiente de correlación Rho de Spearman ambas evaluadas al nivel de significancia del $5 \%$. Se concluyó que existe relación significativa $(p<0,05)$ entre las competencias gerenciales y los factores que limitan el crecimiento empresarial de las MYPES agroexportadoras de aceituna y derivados de Tacna en el año 2018. Aunque presentan una asociación positiva media (rho= 0,543), donde de manera mayoritaria el 58,33 \% considera que aplica "casi siempre" las diferentes competencias gerenciales para el manejo empresarial mientras que el 41,67\% indica que "siempre" considera a la competencia gerencial para la conducción y decisiones empresariales.
\end{abstract}

\footnotetext{
${ }^{1}$ Maestro en Administración y Dirección de Empresas, Tacna, Perú

${ }^{2}$ Doctor en Ciencias Ambientales, Docente Universidad Privada de Tacna, Tacna, Perú
} 
Palabras clave: Competencias gerenciales, crecimiento empresarial.

\begin{abstract}
Determine the relationship that exists between the managerial competencies of the entrepreneurs and the factors that limit the business growth of the agro-export MYPES of olive and Tacna derivatives. The research is crosssectional correlation. According to the researcher's interference in the study, it is a non-experimental investigation. The population is limited to the MYPE entrepreneurs dedicated to the agro-exporter sector of olives and derivatives of the city of Tacna, the sample size selected applying the non-probabilistic sampling. The data processing involved Frequency tables, bar graphs, chi square association tests and Spearman's Rho correlation coefficient both evaluated at the $5 \%$ significance level. It was concluded that there is a significant relationship $(p<0.05)$ between managerial competencies and the factors that limit the business growth of the agro-export MYPES of olive and Tacna derivatives in 2018. Although they present an average positive association ( $r$ o $=0.543$ ), where a majority of $58.33 \%$ consider that the different managerial competencies for business management apply "almost always" while $41.67 \%$ indicate that they "always" consider management competence for driving and business decisions.
\end{abstract}

Keywords: Managerial competences, business growth..

\title{
INTRODUCCIÓN
}

En la actualidad, "Las Micro y Pequeñas empresas en el Perú (MYPES), tienen una indiscutible importancia en la economía nacional, por su papel fundamental en la dinámica del mercado, produciendo y ofertando bienes y servicios con valor añadido y contribuyendo a la generación del empleo en la zona donde se desarrollan. En Tacna, las MYPES agroindustriales tienen gran movimiento comercial debido a las exportaciones de productos como: aceituna, aceite de olivo, orégano, etc. Si bien, las MYPES representan un papel muy importante en la economía peruana, el terreno donde se desarrolla todavía es muy frágil, pues se observan bajos índices de supervivencia y consolidación empresarial de las mismas, muchos de ellos no superan los cinco años de funcionamiento» (Avolio, et al., 2011). En el 2014, "La producción de aceitunas en el país fue de 115,201 toneladas, de las cuales 37,731 se destinaron a la exportación. Pero, a partir del año 2015, tanto la producción como la exportación de este fruto sufrieron "una caída sensible debido a los efectos del Fenómeno El Niño" y por la gran producción egipcia, que empujó los precios hacia abajo, según explicó Morales. El peor año fue el 2016 , en el que la producción se redujo hasta 27,737 toneladas, de las cuales 24,868 toneladas fueron exportadas por un valor de US\$32,585,030. No obstante, el año 2018 presentó una significativa mejoría para el sector: la producción alcanzó las 150,000 toneladas, y se exportaron 27,725 toneladas por US\$31,750,146» (Rosales, 2019). Los dos principales países compradores de aceituna peruana son Chile (53\% de los envíos peruanos) y Brasil (39\%). Miranda (2005) al analizar detalladamente la situación 
económica y comercial de las MYPES en Perú «determinó que la ciudad de Tacna es la segunda, luego de Lima, con las MYPES de mayor capacidad exportadora a nivel nacional con 68 MYPES exportadoras representando el 3,7\% del total nacional».

López (2014) señala, que "Una competencia es la capacidad que tiene una persona de poner en práctica de manera completa sus conocimientos, habilidades, experiencias y características personales en un puesto de trabajo determinado". Para Alles (2009) siguiendo a Spencer y Spencer, afirma "que las competencias son, en definitiva, características fundamentales del hombre e indican "formas de comportamiento o de pensar, que generalizan diferentes situaciones y duran por un largo período de tiempo". Benavides (2002) señala al respecto, que "las competencias pueden distinguirse como comportamientos manifiestos en el desempeño laboral que le permiten a una persona actuar eficazmente». Mondy y Noe (2005) las competencias laborales (gerenciales), pueden definirse como «la capacidad para resolver un problema en una situación dada. En otras palabras, implica la capacidad de movilizar una serie de atributos para trabajar exitosamente». Echevarría (2002) menciona sobre las competencias gerenciales: "Comportamientos competitivos específicos, frente a productos, servicios, ventas o posicionamiento, que hace referencia al uso y mejoramiento del desempeño personal en el manejo de recursos financieros tecnológicos, de información, físicos, de sistemas y a la consolidación de duraderas relaciones interpersonales delegadas" (p.40).

Diferentes trabajos examinados abordan esta teoría bajo diferentes enfoques según la disciplina o el punto de vista adoptado por el autor, de ahí que los autores Acosta y González (2001) afirman que «el crecimiento empresarial no cuenta con una teoría general ni con una definición conceptual generalmente aceptada, pues dicho concepto se rige a distintos enfoques, parámetros de medida y factores determinantes». Para Correa, Rodríguez «el crecimiento empresarial supone no sólo un incremento de las magnitudes económicas, sino también de la complejidad de la organización empresarial, surgiendo una cierta polémica sobre si es el crecimiento el que condiciona la dimensión o, si es la búsqueda de una determinada dimensión óptima la que condiciona el crecimiento»

En la agroindustria participan una gran diversidad de actores que interactúan a partir de relaciones económicas, sociales e institucionales. Se incluyen los actores directamente involucrados en las fases del ciclo productivo, y aquellos que tienen alguna competencia asistencial, política, o financiera» (Fletes, 2006; Grass, 2011). José Menza, Servio Enríquez, Jorge Dorado, José Villarreal, Carlos López y Laura Rojas (2003), establecen que se presentan factores socioeconómicos que limitan u obstaculizan el desarrollo empresarial. Hernández, Silvestri, Añez y Gamboa (2008), establece el perfil de competencias gerenciales de los directivos de las PyME venezolanas, llegó a la conclusión de que el mismo está conformado por una serie de indicadores como los conocimientos, habilidades, rasgos y temperamento motivación y necesidades, capacidad de adecuación, generación y aplicación. Avolio, Mesones y Roca (2011), identificaron factores, administrativos, operativos, estratégicos, externos y personales, los cuales limitan la consolidación y el crecimiento de los empresarios MYPES. Arrincón 
(2005) destaca que las deficiencias estructurales que limitan el desarrollo de las PYMES: la informalidad e improvisación del personal que labora en el establecimiento, falta de capacitación, gestión deficiente, dificultad de acceso a financiamiento, falta de planificación y programas. Miranda (2005) establece lineamientos estratégicos para promover el desarrollo de las PYMES mediante el fomento de la productividad y competitividad, fomentar la cultura del emprendimiento, desarrollar y desplegar tecnologías de información y comunicaciones. Marín (2018) determinaron que la gestión de la innovación influye significativamente en la competitividad en las empresas agroindustriales. Pilco (2018) concluye que existe una relación positiva entre la gestión empresarial y la competitividad en el trabajo de Pymes.

Porter (2009, p.163) lo define como "La capacidad que tiene una organización, pública o privada, lucrativa o no, de obtener y mantener ventajas comparativas que le permitan alcanzar, sostener y mejorar una determinada posición en el entorno socioeconómico". Las competencias gerenciales según Hellriegel (2002) «están conformadas por el conjunto de conocimientos, destrezas, comportamientos y aptitudes que necesita una persona para ser eficiente en una amplia gama de labores gerenciales y en diversas organizaciones.

\section{OBJETIVOS}

Establecer la relación que existe entre las capacidades estratégicas, eficacia personal e innovación y los factores que limitan el crecimiento empresarial de las MYPES agroexportadoras de aceituna y derivados de Tacna en el año 2018.

\section{METODOLOGÍA}

La investigación es aplicada de nivel relacional, no experimental de corte transversal. La población está delimitada a los empresarios MYPE dedicadas al rubro agroindustrial de la provincia de Tacna. La muestra fue a conveniencia conformada por 24 gerentes de las empresas exportadoras agroindustriales del rubro aceituna de la provincia de Tacna, año 2018. Para validar el instrumento se realizó una prueba piloto Instrumentos para la recolección de los datos. El cuestionario estuvo en escala de Likert y fue sometido a la prueba de validez Alpha de Cronbach (0.80). Se utilizó el coeficiente de correlación Rho de Spearman para efectuar el contraste

\section{RESULTADOS}

En la Tabla 2, del 100\% de los gerentes encuestados el 33,3\% afirma que siempre aplican estrategias en su toma de decisiones, mientras que un $29,17 \%$ indica que a veces aplican estrategias mientras que el $37,50 \%$ indica que casi siempre aplican estrategias. En la Tabla 3, del 100\% de los gerentes encuestados el 50,00 \% afirma que siempre aplica intrategias en su toma de decisiones, mientras que un 45,83 \% indica que a casi siempre aplican intrategias y apenas un 4,17\% afirma que a veces aplican intrategias. 
El 50,00 \% afirma que siempre aplica eficacia profesional en su toma de decisiones, mientras que un $41,67 \%$ indica que casi siempre considera la eficacia personal y un 8,33 $\%$ indica que a veces buscan la eficacia personal (Tabla 4). El 45,83\% afirma que "casi siempre" aplican la innovación en sus actividades empresariales, mientras que un 45,83\% (Tabla 5). El 66,67 \% afirma que "siempre" los aspectos administrativos en sus actividades empresariales limitan su desarrollo; (Tabla 6). El 75\% afirma que "siempre" los aspectos administrativos en sus actividades empresariales limitan su desarrollo; mientras que un 20,83\% indica que "casi siempre (Tabla 7). El 50,00 \% afirma que "siempre" los aspectos estratégicos de sus actividades empresariales limitan su desarrollo; mientras que un 41,67 \% indica que "casi siempre" (Tabla 8). El 36,84\% afirma que "A veces" los aspectos externos afectan sus actividades empresariales limitando su desarrollo; mientras que un 31,58 \% indica que "casi siempre" y "siempre" (Tabla 9). El 52,63\% refiere que "Casi siempre" las competencias gerenciales afectan sus actividades empresariales limitan su desarrollo; mientras que un 47,37 \% indica que "siempre" (Tabla 10). El 58,33 \% afirma que "siempre" buscan el crecimiento empresarial en sus actividades empresariales; mientras que un $37,50 \%$ indica que "casi siempre" (Tabla 11)

Tabla 1. Distribución por sexo, edad y nivel de instrucción de los encuestados

\begin{tabular}{lcc}
\hline & $\mathbf{n}$ & $\%$ \\
\hline Sexo & & \\
Masculino & 14 & 58,3 \\
Femenino & 10 & 41,7 \\
Total & 24 & 100,0 \\
\hline Edad & & \\
30 a menos & 4 & 16,7 \\
31 a 34 & 6 & 25,0 \\
35 a 44 & 7 & 29,2 \\
45 a más & 7 & 29,2 \\
Total & 24 & 100,0 \\
\hline Grado de instrucción & \\
Secundario & 5 & 20,8 \\
Técnica & 2 & 8,3 \\
Superior & 16 & 66,7 \\
Total & 24 & 100,0 \\
\hline
\end{tabular}

Tabla 2. Distribución de la dimensión competencia gerencial estratégica

\begin{tabular}{lcc}
\hline Estratégica & $\mathbf{n}$ & $\mathbf{\%}$ \\
\hline A veces & 7 & 29,2 \\
Casi siempre & 9 & 37,5 \\
Siempre & 8 & 33,3 \\
Total & 24 & 100,0 \\
\hline
\end{tabular}

Tabla 3. Distribución de la dimensión competencia gerencial intratégica

\begin{tabular}{lcc}
\hline Intratégica & $\mathbf{n}$ & $\%$ \\
\hline A veces & 1 & 4,2 \\
Casi siempre & 11 & 45,8 \\
Siempre & 12 & 50,0 \\
Total & 24 & 100,0 \\
\hline
\end{tabular}

Tabla 4. Distribución de la dimensión competencia gerencial eficacia personal

\begin{tabular}{lcc}
\hline Eficacia personal & $\mathbf{n}$ & $\mathbf{\%}$ \\
\hline A veces & 2 & 8,3 \\
Casi siempre & 12 & 50,0 \\
Siempre & 10 & 41,7 \\
Total & 24 & 100,0 \\
\hline
\end{tabular}

Tabla 1. Distribución de la dimensión competencia gerencial: Innovación

\begin{tabular}{lcc}
\hline Innovación & $\mathbf{n}$ & $\%$ \\
\hline A veces & 2 & 8,3 \\
Casi siempre & 11 & 45,8 \\
Siempre & 11 & 45,8 \\
Total & 24 & 100,0 \\
\hline
\end{tabular}


Tabla 6. Distribución del factor limitante de tipo administrativo

\begin{tabular}{lcc} 
& $\mathbf{n}$ & $\mathbf{\%}$ \\
\hline A veces & 3 & 12,5 \\
Casi siempre & 5 & 20,8 \\
Siempre & 16 & 66,7 \\
Total & 24 & 100,0 \\
\hline
\end{tabular}

Tabla 2. Distribución del factor limitante de tipo operativos

\begin{tabular}{lcc} 
& $\mathbf{n}$ & $\mathbf{\%}$ \\
\hline A veces & 1 & 4,2 \\
Casi siempre & 5 & 20,8 \\
Siempre & 18 & 75,0 \\
Total & 24 & 100,0 \\
\hline
\end{tabular}

Tabla 3. Distribución del factor limitante de tipo estratégicos

\begin{tabular}{lcc}
\hline & $\mathbf{n}$ & $\mathbf{\%}$ \\
\hline A veces & 2 & 8,3 \\
Casi siempre & 10 & 41,7 \\
Siempre & 12 & 50,0 \\
Total & 24 & 100,0 \\
\hline
\end{tabular}

Tabla 9. Distribución del factor limitante de tipo externo

\begin{tabular}{lcc}
\hline & $\mathbf{n}$ & $\mathbf{\%}$ \\
\hline Nunca & 1 & 4,2 \\
A veces & 9 & 37,5 \\
Casi siempre & 8 & 33,3 \\
Siempre & 6 & 25,0 \\
Total & 24 & 100,0 \\
\hline
\end{tabular}

Tabla 10. Competencias gerenciales

\begin{tabular}{lcc}
\hline & $\mathbf{n}$ & $\mathbf{\%}$ \\
\hline Casi siempre & 14 & 58,3 \\
Siempre & 10 & 41,7 \\
Total & 24 & 100,0
\end{tabular}

Tabla 11. Crecimiento empresarial

\begin{tabular}{lcc}
\hline & $\mathbf{n}$ & $\mathbf{\%}$ \\
\hline A veces & 1 & 4,2 \\
Casi siempre & 9 & 37,5 \\
Siempre & 14 & 58,3 \\
Total & 24 & 100,0 \\
\hline
\end{tabular}

El análisis de normalidad muestra que tanto los indicadores y las variables siguen un comportamiento no paramétrico y por lo tanto para las respectivas pruebas de hipótesis se justifica la aplicación de la prueba de correlación de Spearman para evaluar las relaciones propuestas.

Existe relación significativa entre las competencias gerenciales de los empresarios y los factores que limitan el crecimiento empresarial de las MYPES.

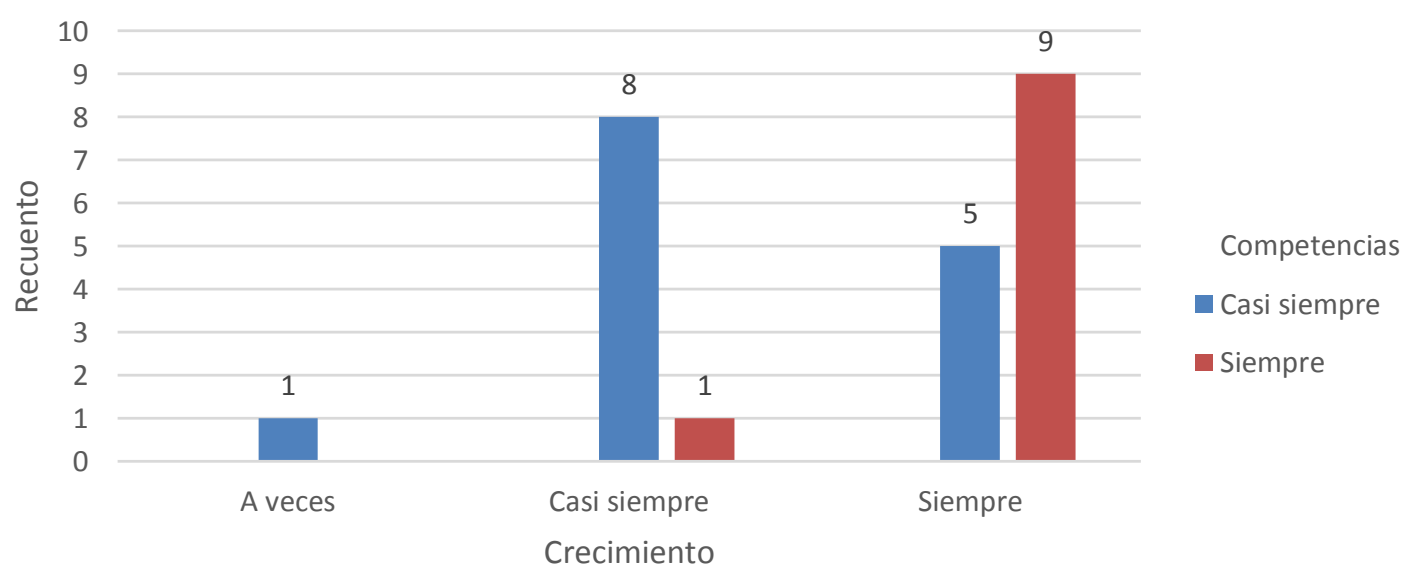


Figura 11. Las competencias gerenciales y los factores que limitan el crecimiento empresarial

No existe una asociación significativa entre las capacidades gerenciales y el crecimiento empresarial (p: 0,208), (Rho: 0,354 p: 0,089) Se concluyó que la aparente relación positiva no es significativa. Es decir, que no necesariamente las capacidades gerenciales aplicadas son suficientes para incidir en el desarrollo empresarial de las MYPES (fig.1)

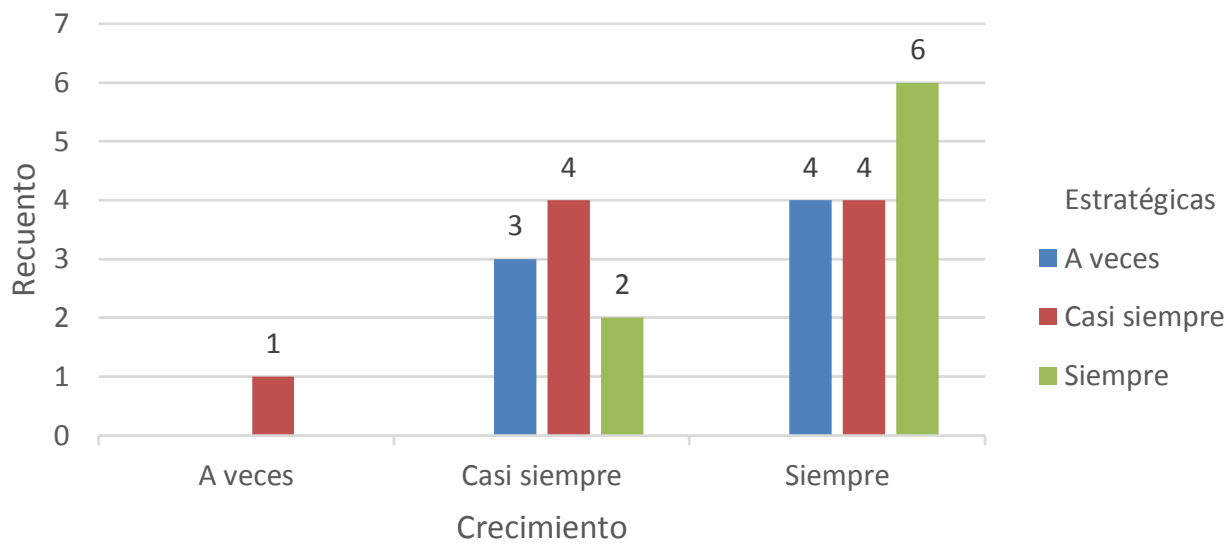

Figura 2. Capacidades estratégicas y los factores limitantes del crecimiento

No existe una asociación significativa entre las capacidades estratégicas y las limitantes del crecimiento empresarial (p: 0,639); (Rho: 0,150 p: 0,485) Se concluyó que la aparente relación positiva no es significativa. Es decir que la aplicación de estrategias en la gestión agroindustrial olivícola no necesariamente incide en su desarrollo empresarial de las MYPES (fig.2)

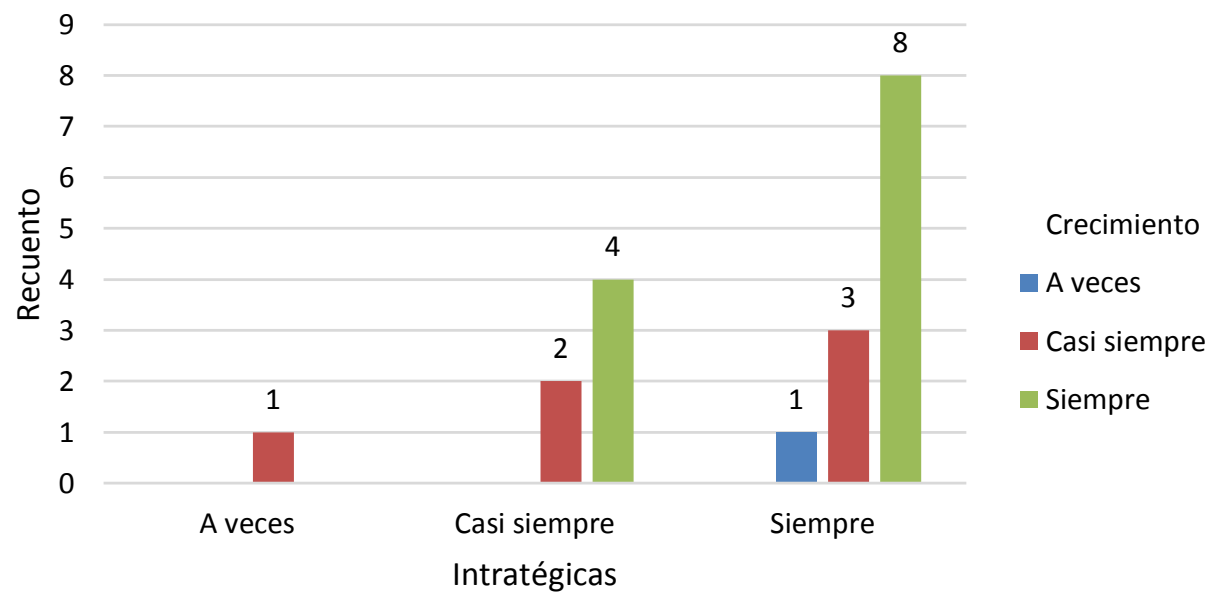

Figura 3. Capacidades intratégicas y los factores que limitan el crecimiento empresarial

No existe una asociación significativa entre las capacidades intratégicas y las limitantes del crecimiento empresarial ( $p: 0,574)$, (Rho: 0,162 p: 0,449) Se concluyó que la aparente relación positiva no es significativa. Es decir que la aplicación de intrategias en la gestión 
agroindustrial olivícola no necesariamente incide en su desarrollo empresarial de las MYPES. (fig.3)

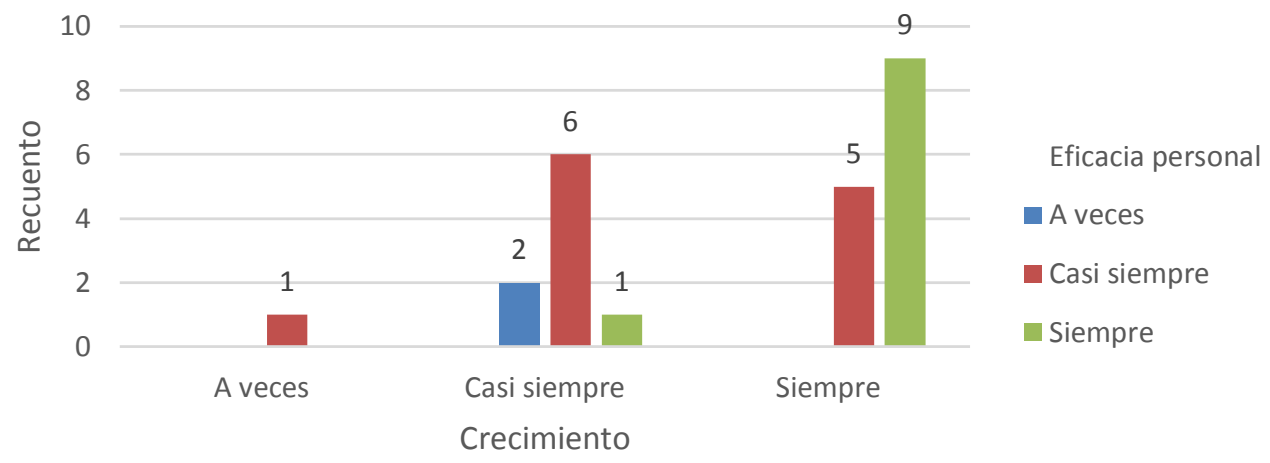

Figura 4. La eficacia personal y los factores que limitan el crecimiento empresarial

No existe una asociación significativa entre la eficacia personal y el crecimiento empresarial ( $p: 0,141$ ), (Rho: 0,466 : 0,022) La aparente relación positiva si es significativa. (fig.4)

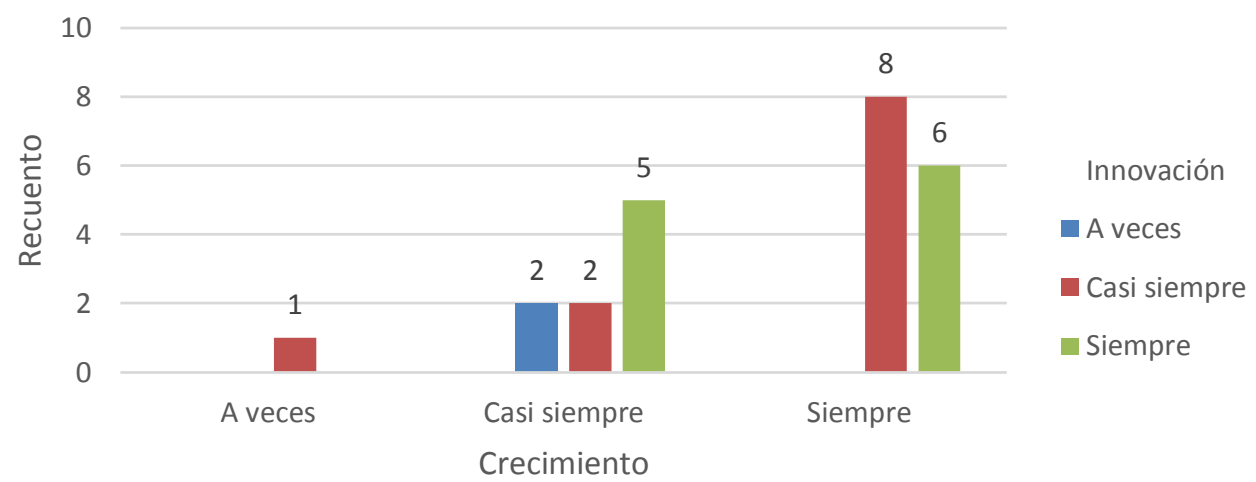

Figura 5. La innovación y los factores que limitan el crecimiento empresarial

No existe una asociación significativa entre la capacidad de innovación y el crecimiento empresarial ( $p: 0,19)$, (Rho: 0,064 p: 0,768) Se concluyó que la aparente relación positiva no es significativa. Es decir, que el incidir en la innovación como método de capacidad gerencial en la gestión agroindustrial olivícola no necesariamente está en relación directa con su desarrollo empresarial de las MYPES.

\section{DISCUSIÓN}

La innovación es la modificación de uno o varios aspectos de la empresa (cambio, desarrollo, transformación, progreso) que tienen como objetivo la creación de nuevos productos, procesos, servicios o valores. "Dado el entorno dinámico al que se hizo referencia anteriormente donde existen cambios sociales, tecnológicos, culturales, etc., las organizaciones no pueden ser ajenas a ellos, por lo que el planteamiento de las organizaciones actuales no debe limitarse a la sostenibilidad sino a la capacidad para enfrentarse y adaptarse a dicho dinamismo con sustentabilidad» (Zapata, 2014). 
Actualmente en el rubro de aceitunas y derivados las exigencias sanitarias son tales que no todos los empresarios han conseguido alcanzar los estándares exigidos por la normativa nacional, es decir que aún les cuesta adaptarse y por lo tanto, el adecuarse a las normativas de calidad en vez de ser una fortaleza, se ha convertido en una limitante también para su desarrollo. De acuerdo con Bunk (1994) posee competencia profesional quien dispone de los conocimientos, destrezas y aptitudes necesarias para ejercer una profesión, resuelve problemas profesionales de forma autónoma y flexible, y está capacitado para colaborar en su entorno profesional y en la organización del trabajo. "Las empresas, estas requieren del personal con capacidades según sus necesidades empresariales, necesidades que van de la mano con el costo que implica disponer de dicho personal capacitado, costo que es el con más frecuencia se subvalora, y se da preferencia por cumplir con las exigencias y costos tributarios que se han convertido en una barrera de crecimiento para la actividad del rubro aceitunas y derivados. Eso explicaría porque ahora casi todas las empresas dedicadas a la agroexportación se hayan trasladado al distrito la Yarada -Los Palos a fin de obviamente, minimizar sus costos». Para Mertens (1996) competencia es la capacidad real para lograr un objetivo o resultado en un contexto dado. "Para la sobrevivencia de las empresas es vital contar con empleados capaces que tengan los conocimientos, habilidades y actitudes requeridas para hacer frente a las transformaciones que ocurren en el mundo. Se requiere de personas con la habilidad para identificar aquello que está cambiando y con la capacidad para dar soluciones a los problemas que enfrentan» (Romero y Rangel, 2008) además Tobón (2006) afirma que «las empresas están cambiando para adaptarse a las dinámicas sociales y económicas, lo que les exige contar con personas que posean un alto grado de flexibilidad para adecuarse a los nuevos puestos y procesos de trabajo, características que aparentemente si bien se aplica pero siempre tomando en cuenta el costo que implica disponer de dicho personal capacitado, es decir siempre el empresario por lo general opta por el profesional u obrero que menores exigencias remunerativas exige, lo que evidentemente no está relacionado con una mayor capacitación, sino todo lo contrario». Además, «la tendencia de las empresas a utilizar offshoring, entendiéndose esto como el proceso que implica la reubicación de las funciones empresariales a un lugar de más bajo costo, y el outsourcing que es el proceso que implica subcontratar el trabajo con un tercero, la globalización que requiere de trabajos más especializados, la movilidad de la fuerza laboral debido a la migración de los países pobres a los países ricos en busca de oportunidades laborales, así como profesionistas de empresas trasnacionales que se mudan de un país a otro debido a las exigencias laborales, aunado a los cambios tecnológicos, la creciente competencia por los mercados y la exigencia por empresas comprometidas con su medio ambiente» (Manpower, 2006). Todos esos cambios tienen como meta ahorrar costos y no necesariamente elevar la calidad de en la productividad empresarial, lo que ocasiona que dicho rubro no requiera de personal preparado y/o que los que existen sean subvalorados en su capacidad. "El Perú no cuenta con suficientes recursos humanos debidamente preparados, no existe a nivel nacional mecanismos de incentivos para la investigación y el desarrollo de tecnología adaptada para el sector productivo, pero existe una dependencia tecnológica con otros países. Las MYPES en el Perú, no cuentan con el respaldo de una infraestructura especializada ni de un sistema de logística que 
permita minimizar los costos de transporte y los tiempos de entrega, así como un acceso limitado al crédito, no contando con tasas competitivas como si se tienen en otros países de la región» (Espino, 2005). Los gerentes agroexportadores de aceitunas y derivados deben fortalecer sus competencias gerenciales pues el problema mayor que enfrentan las MYPES es la falta de educación, visión y sentido de cooperación. Deben consolidar sus capacidades intratégicas, pues ello incidirá en una mayor actitud que fomentará una mayor participación en gremios y alianzas, compitiendo, pero a la vez cooperando. Deben participar en eventos de capacitación de la eficacia personal porque se requiere, ante todo, personal con una base educativa sólida y una profesionalización de la gerencia. Además, deben reforzar sus capacidades de innovación pues, el acceso a las tecnologías de información no constituye necesariamente la solución para las pymes, puesto que inherentemente no les va a proporcionar las mejores prácticas para su negocio. Para la formación de agrupaciones de empresas, que con un interés en común se deben asociar para lograr ventajas que de manera independiente no podrían conseguir, los gerentes agroexportadores de aceitunas y derivados deben participar en eventos de capacitación estratégica.

\section{REFERENCIAS BIBLIOGRÁFICAS}

Acosta M., González A. (2001). Factores determinantes del crecimiento en la Pyme canaria: Influencia del tamaño, la edad y el sector de actividad. Fundación FYDE-Caja Canarias. España.

Alles M. (2009). Dirección estratégica de recursos humanos gestión por competencias: el diccionario Editorial Granica, Buenos Aires. Argentina.

Ansoff, H. I. (1965). Corporate Strategy: An Analytic Approach to Business Police for Growth and Expansion. McGraw-Hill. New York, NY.

Anzizu, J. (1998). El Management ¿Arte o Ciencia? Madrid, España: Fondo Editorial Español.

Arrincón A. (2005). Estrategias y ventajas competitivas para el desarrollo de las PYMES agroindustriales del Perú (Tesis de post grado), Universidad nacional Mayor de San Marcos, Lima-Perú.

Avolio, B., Mesones, A., y Roca, E. (2011). Factores que limitan el crecimiento de las micro y pequeñas empresas en el Perú (MYPES).Strategia, (22), 70-80.

Benavides, O. (2002). Competencias y Competitividad. Diseño para organizaciones latinoamericanas. McGrawHill. Colombia

Blázquez, F., Dorta, J. A. y Verona, M. C. (2006). Concepto, perspectivas y medida del Crecimiento empresarial. Cuadernos de Administración PUJ, 19 (31), 16

Bunk, G.P. (1994) La transmisión de las competencias en ja formación y perfeccionamiento profesionales de la RFA. Revista Europea, Formación Profesional. CEDEFOP.

Carrasco, S. (2005). Metodología de la investigación científica ( $\left.1^{\circ} \mathrm{ed}\right)$. Edit. San Marcos, LimaPerú.

CEPAL, G. FAO (1998) Agroindustria y pequeña agricultura: experiencias y opciones de transformación. Santiago de Chile, CEPAL.

Correa, A. (1999). Factores determinantes del crecimiento empresarial. Tesis de doctorado no publicada, Universidad de la Laguna, España.

Dalziel, M., Cubeiro, J. C. y Fernández, G. (1996). Las competencias: clave para una gestión integrada de los recursos humanos. España: Ediciones Deusto.

Echevarría, B.S. (2002). Gestión de competencia de acción profesional. Revista de investigación educativa vol. 20. Barcelona, España.p.40. 
Espino P. (2005). Estrategia de inserción en la nueva economía para las pymes en el Perú. Universidad Nacional de Trujillo. Trujillo Perú.

Fletes, H. (2006). "Cadenas, redes y actores de la agroindustria en el contexto de la globalización. El aporte de los enfoques contemporáneos del desarrollo regional", Espiral, Estudios sobre Estado y Sociedad, vol. 13, núm. 3, pp. 97-122.

García, A., Rodríguez, R., y Victoria, J. (1988). Análisis del crecimiento sostenible por los distintos sectores empresariales. Documentos de trabajo (Universidad de Oviedo. Facultad de Ciencias Económicas), (3), 3-26.

Grass, J. (2011). "El enfoque de sistemas agroindustriales", Análisis del medio rural latinoamericano, pp. 123-136.

Hellriegel, D.; Jackson, Susan y Slocum, John (2002). Administración: Un Enfoque Basado en Competencias. 9na. Edición. Thomson Editores, S.A. de C.V. México.

Hellriegel, D. (2002). Administración. Un enfoque basado en Competencias. Editorial ESIC. España.

Hernández R., Fernández Collado, C. y Baptista P. (2010). Metodología de la investigación. McGrawHill, 5o edición. México.

Hernández, R., Silvestri, K., Añez, Silenis y Gamboa L. (2008). Realidad de la formación gerencial en las pequeñas y medianas empresas venezolanas. Revista venezolana de Gerencia, 13(41), 105-123. Recuperado el 27 de enero de 2017, de ttp://www.scielo.org.ve/scielo.php?script=sci_arttext\&pid=S1315$99842008000100007 \& \operatorname{lng}=e s \&$ tIng=es.

Lévy-Leboyer, C. (2003). La motivación en la empresa: modelos y estrategias. Gestión 2000.

López, V. (2014). Orientación laboral y promoción de la calidad en formación profesional para el empleo. Editorial ELEARNING S.L. Málaga, España.

Marín C. (2018). Gestión de la innovación y su influencia en la competitividad en las empresas agroindustriales, rubro aceituno de la región Tacna, Año 2016. Universidad Nacional Jorge Basadre Grohmann. Tacna, Perú.

Menza J., Enríquez S., Dorado J., Villarreal J., López C. y Rojas L. (2003). Factores socioeconómicos que limitan o favorecen el desarrollo empresarial en el Municipio De Pasto. Universidad Mariana. Pasto, Colombia.

Mertens, F., Vajo, J. y Skeith, S. (2005). La micro y pequeña empresa en latinoametica. Revista latinoamericana de comercio. B, 109(9).

Mertens, L., Competencia Laboral: sistemas, surgimiento y modelos, Montevideo, CINTERFOR/OIT, 1996.

Ministerio de Industria, Comercio y Turismo (2011). Informe sobre el crecimiento empresarial España: (c) Dirección General de Política de la Pequeña y Mediana Empresa.

Miranda R. (2005). “Competencias claves para la gestión empresarial de las Pymes". Gestión del tercer milenio, Revista de investigación de la facultad de Ciencias Administrativas, UNMSM. Lima-Perú.

Mondy R. y Noe, R. (2005). Administración de recursos humanos. Pearson educación.

Okpara, J., y Wynn, P. (2007). Determinants of small business growth constraints in a subSaharan African economy. SAM advanced management journal, 72(2), 24.

Penrose, E. (1962). Teoría del crecimiento de la empresa, Editorial Aguilar, España.

Pilco J. (2018). Gestión Empresarial y Competitividad en las Asociaciones de Olivareros del Sector de la Yarada - Tacna, 2017. Universidad Privada de Tacna. Tacna, Perú.

Romero, T.y Rangel, B. (2008). Profesionales del siglo 21 Las nuevas Habilidades; Reclutadores de empresas trasnacionales dicen qué características buscan en los recién egresados. Universitarios, Reforma.

Tobón, S. (2006). Formación basada en competencias: pensamiento complejo, diseño curricular y didáctica. Bogotá, Colombia: Ecoc Ediciones. 
Vásquez, M. (2005). Perfil basado en competencias gerenciales de los coordinadores administrativos en instituciones de educación superior. Tesis doctoral. Universidad Dr. Rafael Belloso Chacín. Venezuela.

Zapata D. (2014). La Innovación como habilidad gerencial en los procesos de selección para líderes de alto impacto en las organizaciones. Universidad Militar. Nueva Granada, España. 